\title{
Brevibacterium casei
}

National Cancer Institute

\section{Source}

National Cancer Institute. Brevibacterium casei. NCI Thesaurus. Code C86210.

A species of aerobic, Gram-positive, rod shaped bacteria assigned to the phylum

Actinobacteria. This species is nonmotile, non-spore forming, catalase positive, oxidase negative, does not reduce nitrate, but does hydrolyze gelatin and casein. B. casei has a distinctive cheese smell, is commonly found in milk products and on human skin, but is pathogenic and known to be associated with sepsis, meningitis, and peritonitis. 